\title{
A CLASSIFICATION OF THE FINITE EXTENSIONS OF A MULTIDIMENSIONAL BERNOULLI SHIFT
}

\author{
JANET WHALEN KAMMEYER
}

\begin{abstract}
The finite extensions of a multidimensional Bernoulli shift are classified completely, up to factor isomorphism, and up to isomorphism. If such an extension is weakly mixing then it must be Bernoulli; otherwise, it has a finite rotation factor, which has a Bernoulli complementary algebra. This result is extended to multidimensional Bernoulli flows and Bernoulli shifts of infinite entropy.
\end{abstract}

A $\mathbb{Z}^{n}$-Bernoulli shift, defined on a Lebesgue probability space $(X, \mathscr{A}, \mu)$, is a process $(\mathscr{T}, P)$ where $P$ is a finite partition on $X$ and $\mathscr{T}$ is a group of commuting transformations which describe a $\mathbb{Z}^{n}$-action on $X$, such that $\left\{T_{v} P\right\}_{v \in \mathbb{Z}^{n}}$ forms an independent family of partitions. A finite ( $k$-point) extension $\widehat{\mathscr{T}}$ of such a Bernoulli shift is a skew product of this base process with the action of $S_{k}$, the symmetric group on $k$ symbols. This $\widehat{\mathscr{T}}$ is a $\mathbb{Z}^{n}$-action on $X \times\{1, \ldots, k\}$. These definitions will be made precise; but, first, we state the classification theorem and outline the classification.

We will prove the following theorem.

Classification Theorem. Any finite extension of a $\mathbb{Z}^{n}$-Bernoulli shift either is itself a $\mathbb{Z}^{n}$-Bernoulli shift, or is not weakly mixing, and thus has a finite rotation factor.

Before proceeding with the proof, we apply this theorem and outline the resulting classification. It can be shown that the entropy of the finite extension $\widehat{\mathscr{T}}$ is the same as the entropy of the base Bernoulli shift $\mathscr{T}$. Therefore, by the isomorphism theorem for $\mathbb{Z}^{n}$-Bernoulli shifts [2], any two weakly mixing finite extensions of a given base Bernoulli process will be measurably isomorphic.

It remains to classify the nonweakly mixing finite extensions. This will be done in two ways-up to factor isomorphism and up to isomorphism. Although the details of this classification will follow the proof of the classification theorem, the general idea is outlined below.

To accomplish the first classification, up to factor isomorphism, there is a natural way to further extend a finite extension to a larger $\mathbb{Z}^{n} \times S_{k}$-action. In this case, any two finite extensions are factor isomorphic (i.e. isomorphic by an isomorphism which respects their common Bernoulli factor) if and only if the corresponding $\mathbb{Z}^{n} \times S_{k}$-actions are isomorphic. The classification problem

Received by the editors March 13, 1990 and, in revised form, October 31, 1990.

1980 Mathematics Subject Classification (1985 Revision). Primary 28D15; Secondary 28D20.

This work supported in part by the Naval Academy Research Council and in part by N.S.F. Grant DMS-8902919. 
thus reduces to characterizing the isomorphism classes of the larger $\mathbb{Z}^{n} \times S_{k}$ actions. This characterization reduces to an algebraic problem, in which each isomorphism class corresponds to a certain equivalence class depending on the algebraic structure of $S_{k}$.

The second (and weaker) classification to be discussed distinguishes between isomorphism classes of finite extensions. Although these extensions have a common Bernoulli factor, we will not require that the isomorphism preserve this common factor. In this case, the isomorphism class depends entirely on the structure of the Pinsker algebra of the extension, as it has a Bernoulli complementary algebra.

We proceed with the details.

Let $(X, \mathscr{A}, \mu)$ be a Lebesgue probability space. Let $\mathbf{e}_{1}, \ldots \mathbf{e}_{n}$ denote the $n$ fundamental basis vectors of $\mathbb{Z}^{n}$. Let $\left\{T_{\mathbf{e}_{1}}, \ldots, T_{\mathbf{e}_{n}}\right\}$ be $n$ commuting, measure preserving transformations on $X$. Let $\mathscr{T}=\left\{T_{\mathrm{v}}\right\}_{\mathrm{v} \in \mathbb{Z}^{n}}$ be the group of transformations generated by $\left\{T_{\mathbf{e}_{1}}, \ldots, T_{\mathbf{e}_{n}}\right\}$. We call $(X, \mathscr{A}, \mu), \mathscr{T}$ a $\mathbb{Z}^{n}$-dynamical system.

Let $k \geq 2$ be an integer. A finite ( $k$-point) extension of $(X, \mathscr{A}, \mu), \mathscr{T}$ is the skew product of this base process with an action of $S_{k}$, the symmetric group on $k$ symbols. Specifically, let $\widehat{X}=X \times\{1, \ldots, k\}, \widehat{A}=\mathscr{A} \times 2^{\{1, \ldots, k\}}$, and define $\hat{\mu}$ so that $\hat{\mu}(A \times c)=\mu(A) / k$ for all $A \in \mathscr{A}, c \in\{1, \ldots, k\}$. Let $\mathbf{g}=$ $\left(g_{1}, \ldots, g_{k}\right)$ where each $g_{i}: X \rightarrow S_{k}$ is any measurable map such that for all $i, j$ and for all $x \in X, g_{i}\left(T_{\mathbf{e}_{j}} x\right) g_{j}(x)=g_{j}\left(T_{\mathbf{e}_{i}} x\right) g_{i}(x)$. We call this the cocycle compatibility condition. For $i=1, \ldots, n$, let $T_{\mathbf{e}_{i}}^{g}(x, c)=\left(T_{\mathbf{e}_{i}} x, g_{i}(x)(c)\right)$. Notice that $\left\{T_{\mathbf{e}_{i}}^{g}\right\}_{i=1}^{n}$ is a set of $n$ commuting, measure preserving transformations on $\widehat{X}$. Let $\mathscr{T}^{g}$ be the group of transformations generated by $\left\{T_{\mathbf{e}_{i}}^{g}\right\}_{i=1}^{n}$. We say that $(\widehat{X}, \widehat{\mathscr{A}}, \hat{\mu}), \mathscr{T}^{g}$ is a finite $(k$-point) extension of $(X, \mathscr{A}, \mu)$, $\mathscr{T}$.

The approach used to prove the classification theorem is an extension of the approach used by Dan Rudolph [3, 5]. Rudolph's techniques have been modified to apply to $\mathbb{Z}^{n}$-actions, $n \geq 2$. Many of these $\mathbb{Z}^{n}$-techniques were developed by the author [1] in proving this theorem in the special case of twopoint extensions. The reader will often be referred to these two publications for details.

Let $(X, \mathscr{A}, \mu), \mathscr{T}$ be a $\mathbb{Z}^{n}$-Bernoulli shift with generating partition $P$. Let $\mathbf{g}=\left(g_{1}, \ldots, g_{n}\right)$ be a cocylce, $g_{i}: X \rightarrow S_{k}$, which is measurable with respect to $P$, so that sets on which $\mathbf{g}$ is constant are unions of sets of $P$.

Let $D=\left\{D_{c}\right\}_{c=1}^{k}, D_{c}=\{(x, c) \mid x \in X\}$ be the partition of $\hat{X}$ into colors. Let $\bar{P}=P \vee D$, where $(P \vee D)(x, c)=\left(P(x), D_{c}\right)$. Given any $(x, c) \in \widehat{X}$, the $P$-name of $x$ and $D(x, c)$ at one point together determine the coloring of the entire point.

Let $\left\{\bigvee_{\mathbf{v} \in R(m)} B_{\mathbf{v}}\right\}_{m}$ be a sequence of partitions of $X$, whose symbols are the same as those of $\left\{\bigvee_{\mathrm{v} \in R(m)} T_{\mathrm{v}} P\right\}_{m}$. Define another sequence of partitions $\left\{\bigvee_{\mathrm{v} \in R(m)} C_{\mathrm{v}}\right\}_{m}$ of $X$ such that

(1) The sets $C_{\mathbf{v}}$ are labeled $\{1(\mathbf{v}), \ldots, k(\mathbf{v})\} \subset\left\{D_{1}, \ldots, D_{k}\right\}$,

(2) $C_{0}$ is arbitrary, and

(3) for $\mathbf{v} \neq 0, x \in c(\mathbf{v})$ iff $x \in \tilde{c}(0)$, and the set to which $x$ belongs in $B_{0}$ has the label of a set in $P$ on which $g_{\mathbf{v}}(x)(\tilde{c}(0))=c(\mathbf{v})$. 
The partition $\bigvee_{\mathrm{v} \in R(m)} C_{\mathrm{v}}$ is called a coloring for $\bigvee_{\mathrm{v} \in R(m)} B_{\mathrm{v}}$. Notice that if $C_{0}=\{1, \ldots, k\}$, then $\bigvee_{\mathrm{v} \in R(m)} T_{\mathrm{v}}^{g}(\bar{P})=\bigvee_{\mathrm{v} \in R(m)} T_{\mathrm{v}} P \vee C_{\mathrm{v}}$ for all $m$.

We want to define an analog to the nesting property $(\mathrm{N})$ used in the classification of two-point extensions. The main generalization in this case is that there are now many ways in which a coloring can "fail to match." In the two-point case, pieces of two colorings were either identical, or were flips of one another. This is more complicated here. We set forth some preliminary definitions necessary for defining the nesting property.

Let $\left\{m_{j}\right\} \rightarrow \infty$. Let $\bigvee_{\mathbf{v} \in R\left(m_{j}\right)} B_{\mathbf{v}, j} \vee C_{\mathbf{v}, j}$ and $\bigvee_{\mathbf{v} \in R\left(m_{j}\right)} B_{\mathbf{v}, j}^{\prime} \vee C_{\mathbf{v}, j}^{\prime}$ be two sequences of partitions, such that $\lim _{j \rightarrow \infty}\left(\bigvee_{\mathbf{v} \in R\left(m_{j}\right)} B_{\mathbf{v}, j}, \bigvee_{\mathbf{v} \in R\left(m_{j}\right)} B_{\mathbf{v}, j}^{\prime}\right)=0$.

Let $\varepsilon>0$. Choose $J(\varepsilon), \delta(\varepsilon)>0, j>J(\varepsilon)$ and any joining $\bigvee_{\mathrm{v} \in R\left(m_{j}\right)} \bar{B}_{\mathbf{v}, j} \vee$ $\bar{C}_{\mathbf{v}, j} \vee \bar{B}_{\mathbf{v}, j}^{\prime} \vee \bar{C}_{\mathbf{v}, j}^{\prime}$, such that $d_{\left(2 m_{j}+1\right)^{n}}\left(\bigvee_{\mathbf{v} \in R\left(m_{j}\right)} \bar{B}_{\mathbf{v}, j}, \bigvee_{\mathbf{v} \in R\left(m_{j}\right)} \bar{B}_{\mathbf{v}, j}^{\prime}\right)<\delta(\varepsilon)$. Call such joining a $\delta(\varepsilon)$-match. Assume for now that $j$ is fixed and drop the extra notation.

Choose maximal sets $E_{1}, \ldots, E_{s}$ which partition $X$, on which $\bar{B}_{\mathrm{v}} / E_{r}=$ $\bar{B}_{\mathbf{v}}^{\prime} / E_{r}$, except on a subset of indices $S(r)=\left\{\mathbf{v}_{1, r}, \ldots, \mathbf{v}_{a(r), r}\right\}$. Given $\alpha$, and large enough $m$, there exists $t>0$ so that $S(r)$ is an $(\alpha, \delta, t)$-subset of $R(m)$, with "bad" set $B(r)$. Label the resulting grid cubes contained entirely in $S(r)^{c}$ by their midpoints, lexicographically, as

$$
\mathscr{G}(r)=\left\{R\left(\mathbf{w}_{1}(r), t\right), \ldots, R\left(\mathbf{w}_{G}(r), t\right)\right\} .
$$

Refine the partition to $\left\{E_{r, i}\right\}_{r=1}^{a}, \underset{i=1}{i(r)}$, on which both $\bar{B}_{\mathbf{v}} \vee \bar{C}_{\mathbf{v}} / E_{r, i}$ and $\bar{B}_{\mathbf{v}}^{\prime} \vee$ $\bar{C}_{\mathbf{v}}^{\prime} / E_{r, i}$ consist of a single set, for all $\mathbf{v} \in S(r) \cup B(r)$ and all $\mathbf{v}=\mathbf{w}_{j}(r)$, $j=1, \ldots, G$. Further refine the partition to $\left\{E_{r, i, j}\right\}_{r=1}^{a}, \underset{i=1}{i(r)}, \underset{j=1}{j(i, r)}$ so that for all $\mathbf{v} \in S(r)$, both $g_{\mathbf{v}}(x)$ and $g_{\mathbf{v}}^{\prime}(x)$ are single elements of $S_{k}$, for all $x \in E_{r, i, j}$. Let $F_{1}, \ldots, F_{s}$ be a listing of this partition $\left\{E_{r, i, j}\right\}$. Note that for all $\mathbf{v} \in R(m), g_{\mathbf{v}}^{\prime}(x)^{-1} \circ g_{\mathbf{v}}(x)=\sigma_{\mathbf{v}, r}$ is a single element of $S_{k}$, for all $x \in F_{r}$.

For each $r$, define a partition of $R(m)$ given by $\{S(r, \sigma)\}_{\sigma \in S_{k}}$ so that $\mathbf{v} \in$ $S(r, \sigma)$ if and only if $\sigma_{\mathbf{v}, r}=\sigma$. Note that each $R\left(\mathbf{w}_{j}(r), t\right)$ in $\mathscr{G}(r)$ lies in exactly one $S(r, \sigma)$.

We say that $\left\{F_{r},\{S(r, \sigma)\}_{\sigma \in S_{k}}\right\}_{r=1}^{s}$ is a blocking of the joining. Let $\mathscr{M}(r)=$ $\left\{\mathbf{v} \in R(m) \mid \bar{C}_{\mathbf{v}} / F_{r}=\bar{C}_{\mathbf{v}}^{\prime} / F_{r}\right\}$, so that $\mathscr{M}(r)$ is a union of some subset of the $S(r, \sigma)$. In the case of a two-point extension, this union is always a single set, $\sigma=$ identity. In general, however, this will be a finite union, since different elements of $S_{k}$ could fix the same color. Think of $\mathscr{M}(r)$ as the "places" where the two colorings match. Note that if $\mathbf{w}_{j}(r) \in \mathscr{M}(r)$, then the entire block $R\left(\mathbf{w}_{j}(r), t\right) \subseteq \mathscr{M}(r)$.

A monochrome coloring $\bigvee_{\mathrm{v} \in R(m)} C_{\mathbf{v}}$ for $\bigvee_{\mathrm{v} \in R(m)} B_{\mathbf{v}}$ is one for which $C_{\mathbf{v}}$ is $\bigvee_{\mathbf{v} \in R(m)} B_{\mathbf{v}}$-measurable.

Finally, in order to define the nesting property, we must have a way to discuss the closeness of colored distributions. Two colored sequences $\bigvee_{\mathrm{v} \in R(m)} B_{\mathbf{v}} \vee$ $C_{\mathrm{v}}$ and $\bigvee_{\mathrm{v} \in R(m)} B_{\mathrm{v}}^{\prime} \vee C_{\mathrm{v}}^{\prime}$ are $(\varepsilon, a)$-close if there exists a joining such that $d_{(2 m+1)^{n}}\left(\bigvee_{\mathrm{v} \in R(m)} \bar{B}_{\mathrm{v}}, \bigvee_{\mathrm{v} \in R(m)} \bar{B}_{\mathrm{v}}^{\prime}\right)<\varepsilon$ and $d_{(2 m+1)^{n}}\left(\bigvee_{\mathrm{v} \in R(m)} \bar{C}_{\mathbf{v}}, \bigvee_{\mathrm{v} \in R(m)} \bar{C}_{\mathrm{v}}^{\prime}\right)<$ $a$. These sequences are $(\varepsilon, a)$-rigidly apart if any joining with 


$$
d_{(2 m+1)^{n}}\left(\bigvee_{\mathbf{v} \in R(m)} \bar{B}_{\mathbf{v}}, \bigvee_{\mathbf{v} \in R(m)} \bar{B}_{\mathbf{v}}^{\prime}\right)<\varepsilon
$$

also satisfies $d_{(2 m+1)^{n}}\left(\bigvee_{\mathbf{v} \in R(m)} \bar{C}_{\mathbf{v}}, \bigvee_{\mathbf{v} \in R(m)} \bar{C}_{\mathbf{v}}^{\prime}\right)>1-a$. We are now prepared to define "nesting."

Any two sequences $\bigvee_{\mathbf{v} \in R\left(m_{j}\right)} B_{\mathbf{v}, j} \vee C_{\mathbf{v}, j}$ and $\bigvee_{\mathbf{v} \in R\left(m_{j}\right)} B_{\mathbf{v}, j}^{\prime} \vee C_{\mathbf{v}, j}^{\prime}$ are said to nest (to $\bar{\varepsilon}$ ) with rate $A, \delta(\cdot)$ if $\forall \varepsilon>0, \exists J(\varepsilon), \delta(\varepsilon)$ (as above) and $\bar{\varepsilon}(\varepsilon)<\varepsilon$, such that either (i) for at least $1-\bar{\varepsilon} / 2$, in measure, of the $F_{r},|\mathscr{M}(r)| \geq$ $(1-\bar{\varepsilon} / 2)(2 m+1)^{n}$, or (ii) for at least $A$, in measure, of $F_{r}$, with $|\mathscr{M}(r)|<$ $(1-\bar{\varepsilon} / 2)(2 m+1)^{n}$, for at least $A$, in density of the "blocks" $S(r, \sigma) \notin \mathscr{M}(r)$, for at least $A$, in measure, of the atoms $E$ of $\bigvee_{\mathbf{v} \in \mathscr{M}(r)} \bar{B}_{\mathbf{v}, j} \vee \bar{C}_{\mathbf{v}, j} \vee \bar{B}_{\mathbf{v}, j}^{\prime} \vee \bar{C}_{\mathbf{v}, j}^{\prime}$, $\bigvee_{\mathbf{v} \in S(r, \sigma)} \bar{B}_{\mathbf{v}, j} \vee \bar{C}_{\mathbf{v}, j} / F_{r} \cap E$ and $\bigvee_{\mathbf{v} \in S(r, \sigma)} \bar{B}_{\mathbf{v}, j}^{\prime} \vee \bar{C}_{\mathbf{v}, j}^{\prime} / F_{r} \cap E$ are $(\varepsilon, 1-A)$-close.

Lemma 1. Any distributions $\bigvee_{\mathbf{v} \in R\left(m_{j}\right)} B_{\mathbf{v}, j} \vee C_{\mathbf{v}, j}$ and $\bigvee_{\mathbf{v} \in R\left(m_{j}\right)} B_{\mathbf{v}, j}^{\prime} \vee C_{\mathbf{v}, j}^{\prime}$ which nest at rate $A, \delta(\cdot)$ must satisfy

$$
\lim _{j \rightarrow \infty} \bar{d}_{\left(2 m_{j}+1\right)^{n}}\left(\bigvee_{\mathbf{v} \in R\left(m_{j}\right)} B_{\mathbf{v}, j} \vee C_{\mathbf{v}, j}, \bigvee_{\mathbf{v} \in R\left(m_{j}\right)} B_{\mathbf{v}, j}^{\prime} \vee C_{\mathbf{v}, j}^{\prime}\right)=0 .
$$

Proof. Let $\varepsilon>0$. Choose $J(\varepsilon), \delta(\varepsilon)$, so that for $j>J(\varepsilon)$,

$$
\bar{d}_{\left(2 m_{j}+1\right)^{n}}\left(\bigvee_{\mathbf{v} \in R\left(m_{j}\right)} B_{\mathbf{v}, j}, \bigvee_{\mathbf{v} \in R\left(m_{j}\right)} B_{\mathbf{v}, j}^{\prime}\right)<\delta(\varepsilon) .
$$

Fix $j$, and drop its notation.

If (i) in the definition of nesting holds, the lemma is proven. Suppose, instead that (ii) in the definition of nesting holds.

Choose $B$ so that $d_{(2 m+1)^{n}}\left(\bigvee_{\mathrm{v} \in R(m)} C_{\mathrm{v}}, \bigvee_{\mathrm{v} \in R(m)} C_{\mathrm{v}}^{\prime}\right) \leq B$.

For each $r$, there must exist some $S\left(r, \sigma_{r}\right)$ which is "good" according to (ii) in the definition of nesting, $S\left(r, \sigma_{r}\right) \notin \mathscr{M}(r)$, such that $\left|S\left(r, \sigma_{r}\right)\right|>$ $(2 m+1)^{n} A /(k !)^{2}$. For each such $r$ and $S\left(r, \sigma_{r}\right)$, for at least $A$, in measure of the atoms, $E \in \bigvee_{\mathbf{v} \in \mathscr{M}(r)} \bar{B}_{\mathbf{v}, j} \vee \bar{C}_{\mathbf{v}, j} \vee \bar{B}_{\mathbf{v}, j}^{\prime} \vee \bar{C}_{\mathbf{v}, j}^{\prime}$, there exists a joining $\bigvee_{\mathbf{v} \in S\left(r, \sigma_{r}\right)} \widetilde{B}_{\mathbf{v}, j, r, E} \vee \widetilde{C}_{\mathbf{v}, j, r, E} \vee \widetilde{B}_{\mathbf{v}, j, r, E}^{\prime} \vee \widetilde{C}_{\mathbf{v}, j, r, E}^{\prime}$ of $\bigvee_{\mathbf{v} \in S(r, \sigma)} \bar{B}_{\mathbf{v}, j} \vee \bar{C}_{\mathbf{v}, j} / F_{r} \cap E$ and $\bigvee_{\mathbf{v} \in S^{\prime}(r, \sigma)} \bar{B}_{\mathbf{v}, j}^{\prime} \vee \bar{C}_{\mathbf{v}, j}^{\prime} / F_{r} \cap E$ with

$$
d_{\left|S\left(r, \sigma_{r}\right)\right|}\left(\bigvee_{\mathbf{v} \in S\left(r, \sigma_{r}\right)} \widetilde{B}_{\mathbf{v}, j, r, E}, \bigvee_{\mathbf{v} \in S\left(r, \sigma_{r}\right)} \widetilde{B}_{\mathbf{v}, j, r, E}^{\prime}\right)<\varepsilon
$$

and $d_{\left|S\left(r, \sigma_{r}\right)\right|}\left(\bigvee_{\mathbf{v} \in S\left(r, \sigma_{r}\right)} \tilde{C}_{\mathbf{v}, j, r, E}, \bigvee_{\mathbf{v} \in S\left(r, \sigma_{r}\right)} \tilde{C}_{\mathbf{v}, j, r, E}^{\prime}\right)<1-A$.

Modify the partitions $\bigvee_{\mathrm{v} \in R(m)} B_{\mathrm{v}} \vee C_{\mathrm{v}}$ and $\bigvee_{\mathrm{v} \in R(m)} B_{\mathrm{v}}^{\prime} \vee C_{\mathrm{v}}^{\prime}$ on these sets $F_{r} \cap E$, by a copy of $\bigvee_{\mathbf{v} \in S\left(r, \sigma_{r}\right)} \widetilde{B}_{\mathbf{v}, j, r, E} \vee \widetilde{C}_{\mathbf{v}, j, r, E} \vee \widetilde{B}_{\mathbf{v}, j, r, E}^{\prime} \vee \widetilde{C}_{\mathbf{v}, j, r, E}^{\prime}$. Elsewhere do not modify the partitions. Call these new partitions $\bigvee_{\mathbf{v} \in R(m)} \widehat{B}_{\mathbf{v}} \vee \widehat{C}_{\mathbf{v}}$ and $\bigvee_{\mathbf{v} \in R(m)} \widehat{B}_{\mathbf{v}}^{\prime} \vee \widehat{C}_{\mathbf{v}}^{\prime}$. These are clearly copies of $\bigvee_{\mathrm{v} \in R(m)} B_{\mathrm{v}} \vee C_{\mathrm{v}}$ and $\bigvee_{\mathrm{v} \in R(m)} B_{\mathrm{v}}^{\prime} \vee C_{\mathrm{v}}^{\prime}$. Furthermore, given $\varepsilon_{1}$, we may choose $\varepsilon$ so that

$$
d_{(2 m+1)^{n}}\left(\bigvee_{\mathbf{v} \in R(m)} \widehat{B}_{\mathbf{v}}, \underset{\mathbf{v} \in R(m)}{\bigvee} \widehat{B}_{\mathbf{v}}^{\prime}\right)<\frac{\varepsilon_{1}}{2}
$$


and $d_{(2 m+1)^{n}}\left(\bigvee_{\mathrm{v} \in R(m)} \widehat{C}_{\mathrm{v}}, \bigvee_{\mathrm{v} \in R(m)} \widehat{C}_{\mathrm{v}}^{\prime}\right)<B\left(1-A^{3}(1-A) /(k !)^{2}\right)$. Apply this process iteratively $i$ times to see that

$$
d_{(2 m+1)^{n}}\left(\bigvee_{\mathrm{v} \in R(m)} \widehat{C}_{\mathbf{v}}, \bigvee_{\mathbf{v} \in R(m)} \widehat{C}_{\mathbf{v}}^{\prime}\right)<\left(1-\frac{A^{3}(1-A)}{(k !)^{2}}\right)^{i}
$$

Choose $i$ so small that $\left(1-A^{3}(1-A) /(k !)^{2}\right)^{i}<\varepsilon_{1} / 2$, and the lemma is proven.

Let $\left\{\bigvee_{\mathbf{v} \in R\left(m_{j, t}\right)} B_{\mathbf{v}}(j, t)\right\}_{(j, t)},(j, t) \in\left(\mathbb{Z}^{+}\right)^{2}$, be a doubly indexed array of distributions, such that $m_{j, t} \rightarrow \underset{j}{\infty}$ for all fixed $t$, and such that

$$
\lim _{j \rightarrow \infty} \bar{d}_{\left(2 m_{j, t}+1\right)^{n}}\left(\bigvee_{\mathrm{v} \in R\left(m_{j, t}\right)} B_{\mathrm{v}}(j, t), \bigvee_{\mathrm{v} \in R\left(m_{j, t}\right)} T_{\mathrm{v}} P\right)=0
$$

To each element in this array, assign $k$ different monochrome colorings, $\bigvee_{\mathbf{v} \in R\left(m_{j, t}\right)} C_{\mathbf{v}}^{c}(j, t), c=1, \ldots, k$, such that for $c \neq c^{\prime}, \bar{d}\left(C_{0}^{c}(j, t), C_{0}^{c^{\prime}}(j, t)\right)$ $=1$. Call this a disjointly colored array and call the $k$ colorings a disjoint coloring.

Let $\bigvee_{\mathbf{v} \in R\left(m_{j, t}\right)} B_{\mathbf{v}}(j, t) \bigvee \bigvee_{c=1}^{k} C_{\mathbf{v}}^{v}(j, t)$ and $\bigvee_{\mathbf{v} \in R\left(m_{j, t}\right)} B_{\mathbf{v}}^{\prime}(j, t) \bigvee \bigvee_{c^{\prime}=1}^{k} C_{\mathbf{v}}^{\prime c^{\prime}}(j, t)$ be two disjointly colored arrays. Let $\mathscr{C}$ be a collection of ordered paris $\left(c, c^{\prime}\right) \in$ $\{1, \ldots, k\}^{2}$ such that if $\left(c, c^{\prime}\right) \in \mathscr{C}$ then $\bigvee_{\mathrm{v} \in R\left(m_{j, t}\right)} B_{\mathbf{v}}(j, t) \vee C_{\mathrm{v}}^{c}(j, t)$ and $\bigvee_{\mathrm{v} \in R\left(m_{j, t}\right)} B_{\mathrm{v}}^{\prime}(j, t) \vee C_{\mathrm{v}}^{\prime c^{\prime}}(j, t)$ are $(\varepsilon(t), 1 / t)$-rigidly apart, for some $\varepsilon(t)>0$. Notice that $|\mathscr{C}| \leq k^{2}$. The goal is to maximize the size of $\mathscr{C}$. Specifically, we will construct a so-called "maximal arrangement" of a colored distribution. We will see that if any maximal arrangement has $\mathscr{C}=\varnothing$, then the extension is Bernoulli, and furthermore, if $\mathscr{C} \neq \varnothing$ then the extension is not weakly mixing. We proceed with the construction.

Lemma 2. Let $\bigvee_{\mathbf{v} \in R\left(m_{j, l}\right)} B_{\mathbf{v}}(j, t) \vee \bigvee_{c=1}^{k} C_{\mathbf{v}}^{c}(j, t)$ and

$$
\bigvee_{\mathbf{v} \in R\left(m_{j, t}\right)} B_{\mathbf{v}}^{\prime}(j, t) \vee \bigvee_{c^{\prime}=1}^{k} C_{\mathbf{v}}^{\prime c^{\prime}}(j, t)
$$

be two disjointly colored arrays with pairs $\mathscr{C}$ of colors which are $(\varepsilon(t), 1 / t)$ rigidily apart. Let $\left(c, c^{\prime}\right) \notin \mathscr{C}$. Then one of the following two statments must hold:

(i) For any $\varepsilon_{1}>0$, there is some $T$ such that for $t \geq T$, and for any $\varepsilon_{2}>0$, there is some $J$, so that for $j>J, \bigvee_{\mathbf{v} \in R\left(m_{j, t}\right)} B_{\mathbf{v}}(j, t) \vee C_{\mathbf{v}}^{c}(j, t)$ and $\bigvee_{\mathrm{v} \in R\left(m_{j, t}\right)} B_{\mathrm{v}}^{\prime}(j, t) \vee C_{\mathrm{v}}^{\prime c^{\prime}}(j, t)$ are $\left(\varepsilon_{2}, \varepsilon_{1}\right)$-close, or

(ii) $\forall A>0, \exists \bar{\varepsilon}>0$ and an infinite set $T(A)$ such that for $t \in T(A)$, there exists some $\hat{\varepsilon}(t, A)>0$, so that for any $\delta>0$, there is an infinite set $J(A, t, \delta)$ so that for $j \in J(A, t, \delta)$, there is a $\delta$-match

$$
\bigvee_{\mathbf{v} \in R\left(m_{j, t}\right)} \bar{B}_{\mathbf{v}}(j, t) \vee \bar{C}_{\mathbf{v}}^{c}(j, t) \vee \bar{B}_{\mathbf{v}}^{\prime}(j, t) \vee \bar{C}_{\mathbf{v}}^{\prime c^{\prime}}(j, t),
$$

so that, in its blocking, for more than $(1-A) \bar{\varepsilon}$ of the $F_{r}$, for more than $(1-A) \bar{\varepsilon}$ of the $S(r, \sigma) \notin \mathscr{M}(r)$, for more than $(1-A)$, in measure, of the 
atoms $E$ in $\bigvee_{\mathbf{v} \in \mathscr{M}(r)} \bar{B}_{\mathbf{v}}(j, t) \vee \bar{C}_{\mathbf{v}}^{c}(j, t) \vee \bar{B}_{\mathbf{v}}^{\prime}(j, t) \vee \bar{C}_{\mathbf{v}}^{\prime c^{\prime}}(j, t)$, the distributions $\bigvee_{\mathbf{v} \in S(r, \sigma)} \bar{B}_{\mathbf{v}}(j, t) \vee \bar{C}_{\mathbf{v}}^{c}(j, t) / F_{r} \cap E$ and $\bigvee_{\mathbf{v} \in S(r, \sigma)} \bar{B}_{\mathbf{v}}^{\prime}(j, t) \vee \bar{C}_{\mathbf{v}}^{\prime c^{\prime}}(j, t) / F_{r} \cap E$ are $(\hat{\varepsilon}(t, A), A)$-rigidly apart.

Proof. Suppose (i) is false. Then $\forall A>0, \exists \bar{\varepsilon}>0$ and an infinite set $T(A)$ so that for $t \in T(A)$, there is no $\delta(\cdot)$ for which the sequence in $j$ nests to $\bar{\varepsilon}$ with rate $A, \delta(\cdot)$. Specifically, $\exists \hat{\varepsilon}(t, A)$ such that for all $\delta>0$, there is an infinite set $J(A, t, \delta)$ so that for $j \in J(A, t, \delta)$, neither (i) nor (ii) in nesting occurs. Write down the negations of these two statements to see that (ii) in this lemma must hold.

A key point here is that if (ii) of Lemma 2 does NOT hold, then nesting FAILS to occur. Suppose now that (ii) of Lemma 2 is satisfied. We must make both $\bigvee_{\mathbf{v} \in S(r, \sigma)} \bar{B}_{\mathbf{v}}(j, t) \vee \bar{C}_{\mathbf{v}}^{c}(j, t) / F_{r} \cap E$ and $\bigvee_{\mathbf{v} \in S(r, \sigma)} \bar{B}_{\mathbf{v}}^{\prime}(j, t) \vee \bar{C}_{\mathbf{v}}^{\prime c^{\prime}}(j, t) / F_{r} \cap E$ into colored distributions.

Fix $r, \sigma, E$, as above. Choose $\hat{\alpha}, \hat{\delta}, m$ so that $S(r, \sigma)^{c}$ is an $(\hat{\alpha}, \hat{\delta}, m)$ subset of $R\left(m_{j, t}\right)$, [1]. Label all of the grid cubes $R\left(\mathbf{v}_{i}, m\right) \subseteq R\left(m_{j, t}\right)$ lexicographically, $\mathbf{v}_{i}<\mathbf{v}_{i+1}$. In this inherited ordering, list the grid cubes contained entirely in $S(r, \sigma)$ as $\left\{s_{1}, \ldots, s_{B}\right\}$. Let $R_{1}=\{R(0, m)\}$. In general, let $R_{j}=\left\{R(\mathbf{v}, m) \notin \bigcup_{i<j} R_{i} \mid R(\mathbf{v}, m)\right.$ touches a cube in $\left.R_{j-1}\right\}$. Place any ordering on each set $R_{j}$. Use this ordering to impose an ordering on the set of all grid cubes by requiring $R_{j}<R_{j+1}$, for all $j$.

There is some maximal $M<m_{j, t}$ such that the number of grid cube translates of $R(0, m)$ in $R(M)$ is less than or equal to $B$. Color all of the lattice points $\mathbf{v} \in R(M)$ red. In addition, color enough new $R(0, m)$-grid cube translates in $R_{M+1}$ to equal $B$, altogether, in the order described above. The resulting figure will not, in general, be symmetric. Let $\widehat{B}=|S(r, \sigma)|-B(2 m+1)^{n}$. Then $\widehat{B}$ is the number of points $\mathbf{v} \in R\left(m_{j, t}\right)$ which lies in $S(r, \sigma)$, but not in a grid cube that lies entirely in $S(r, \sigma)$. Color $\widehat{B}$ single lattice points about the figure, in any manner, so that the $R(m)$-grid cube translates are filled in order.

Let $Q=Q(S(r, \sigma)) \subset R\left(m_{j, t}\right)$ be this newly constructed "red" set in $\mathbb{Z}^{n}$, $|Q|=|S(r, \sigma)|$. Let $\phi: S(r, \sigma) \rightarrow Q$ be a map defined as follows. For each $i=1, \ldots, B$, let $\phi\left(s_{i}\right)$ be the corresponding grid cube in $Q$, so that $\phi\left(s_{i}\right)<$ $\phi\left(s_{i+1}\right)$, lexicographically. Define $\phi$ on the remaining points of $S(r, \sigma)$ to be any bijection onto the leftover points of $Q$. This is a well-defined method of "pushing together" a conditional distribution over indices in $S(r, \sigma)$ to look like a distribution over indices in $Q$.

Write $S(r, \sigma)=\left\{s_{1}, \ldots, s_{B}, \mathbf{v}_{1}, \ldots, \mathbf{v}_{b}\right\}$, where the $s_{i}$ are grid cubes completely contained in $S(r, \sigma)$ and the $\mathbf{v}_{i}$ are the remaining members of $S(r, \sigma)$. For $\mathbf{v} \in s_{i}$ with $\left\|\mathbf{v}-\mathbf{w}_{i}\right\| \neq m$, let $\bar{B}_{\phi(\mathbf{v})}(r, \sigma, E)=\bar{B}_{\mathbf{v}} / E \cap F_{r}=\bar{B}_{\mathbf{v}}^{\prime} / E \cap F_{r}$. The other $\mathbf{v} \in S(r, \sigma)$ fall into several special categories. For $i=1, \ldots, n$, define the following five sets:

$$
\begin{aligned}
& U^{i}=\left\{\mathbf{v} \in S(r, \sigma) \mid \mathbf{v} \in s_{f}, \phi^{-1}\left(\phi(\mathbf{v})+\mathbf{e}_{i}\right) \in s_{g}, \text { some } f, g\right\}, \\
& V^{i}=\left\{\mathbf{v} \in S(r, \sigma) \mid \mathbf{v} \in s_{f}, \phi^{-1}\left(\phi(\mathbf{v})+\mathbf{e}_{i}\right)=\mathbf{v}_{g} \text {, some } f, g\right\} \text {, } \\
& W^{i}=\left\{\mathbf{v} \in S(r, \sigma) \mid \mathbf{v}=\mathbf{v}_{f}, \phi^{-1}\left(\phi(\mathbf{v})+\mathbf{e}_{i}\right) \in s_{g} \text {, some } f, g\right\} \text {, }
\end{aligned}
$$




$$
\begin{gathered}
X^{i}=\left\{\mathbf{v} \in S(r, \sigma) \mid \mathbf{v}=\mathbf{v}_{f}, \phi^{-1}\left(\phi(\mathbf{v})+\mathbf{e}_{i}\right)=\mathbf{v}_{g}, \text { some } f \neq g\right\}, \\
Y^{i}=\left\{\mathbf{v} \in S(r, \sigma) \mid \phi(\mathbf{v})+\mathbf{e}_{i} \notin Q\right\} .
\end{gathered}
$$

For a fixed $I$, the sets $U^{I}, V^{I}, W^{I}, X^{I}$, and $Y^{I}$ are all pair wise disjoint. We use these sets to define $B_{\phi(\mathbf{v})}(r, \sigma, E)$ for all $\mathbf{v}$.

For each $x \in[0,1], \bar{B}_{\mathbf{v}}(x)$ is assigned an element $g_{\mathbf{v}}(x) \in S_{k}$. Let $\mathbf{a} \rightarrow \mathbf{b}$ denote a path from a to $\mathbf{b}$ in an appropriate $s_{f}$, and let $g_{\mathbf{a} \rightarrow \mathbf{b}}(x)$ denote the element of $S_{k}$ which results from the composition of the $g_{\mathrm{v}}(x)$ along the path. For each $\mathbf{v} \in U^{i}$, choose $\bar{B}_{\phi(\mathbf{v})}(r, \sigma, E)$ so that for all $x \in E \cap F_{r}$,

$$
g_{\phi(\mathbf{v})}(x)=\left[g_{\mathbf{w}_{g} \rightarrow \phi^{-1}\left(\phi(\mathbf{v})+\mathbf{e}_{i}\right)}\right] \circ g_{\mathbf{w}_{g}} \circ g_{\mathbf{w}_{f}}^{-1} \circ\left[g_{\mathbf{w}_{f} \rightarrow \mathbf{v}}\right]^{-1}(x) .
$$

For each $\mathbf{v} \in V^{i}$, choose $\bar{B}_{\phi(\mathbf{v})}(r, \sigma, E)$ so that for all $x \in E \cap F_{r}$, we have

$$
g_{\phi(\mathbf{v})}(x)=g_{\mathbf{v}_{g}} \circ g_{\mathbf{w}_{f}}^{-1} \circ\left[g_{\mathbf{w}_{f} \rightarrow \mathbf{v}}\right]^{-1}(x) .
$$

For each $\mathbf{v} \in W^{1}$, choose $\bar{B}_{\phi(\mathbf{v})}(r, \sigma, E)$ so that for all $x \in E \cap F_{r}$, we have

$$
g_{\phi(\mathbf{v})}(x)=\left[g_{\mathbf{w}_{g} \rightarrow \phi^{-1}\left(\phi(\mathbf{v})+\mathbf{e}_{i}\right)}\right] \circ g_{\mathbf{w}_{g}} \circ g_{\mathbf{v}_{f}}^{-1}(x) .
$$

For each $\mathbf{v} \in X^{i}$, choose $\bar{B}_{\phi(\mathbf{v})}(r, \sigma, E)$ so that for all $x \in E \cap F_{r}$, we have $g_{\phi(\mathbf{v})}(x)=g_{\mathbf{v}_{g}} \circ g_{\mathbf{v}_{f}}^{-1}(x)$. For each $\mathbf{v} \in Y^{i}$, the selection does not matter.

Use the cocycle compatibility conditions to see that

$$
\bar{C}_{\phi(\mathbf{v})}^{c}(r, \sigma, E) \vee \bar{C}_{\phi(\mathbf{v})}^{c^{\prime}}(r, \sigma, E)=\bar{C}_{\mathbf{v}}^{c} \vee \bar{C}_{\mathbf{v}}^{\prime c^{\prime}} / E \cap F_{r},
$$

and that these are both monochrome colorings for $\bigvee_{\mathrm{v} \in S(r, \sigma)} \bar{B}_{\phi(\mathrm{v})}(r, \sigma, E)$. We call this the standard modification of the two conditional distributions with which we began. Notice that the uncolored conditional distributions have been modified only at those indices $\mathbf{v}$ such that $\mathbf{v}+\mathbf{e}_{i}$, for some $i$, is not $S(r, \sigma)$.

Recall that the goal is to modify the given conditional distributions in order to increase the size of the set $\mathscr{C}$ of rigidly apart pairs. We must make sure that in the new coloring, no rigidly apart pairs in $\mathscr{C}$ were lost.

Lemma 3. Suppose for two disjointly colored arrays, there exists an $\varepsilon(t)>0$ and $\left(c, c^{\prime}\right) \in \mathscr{C}$ so that $\bigvee_{\mathrm{v} \in R\left(m_{j, t}\right)} B_{\mathrm{v}}(j, t) \vee C_{\mathrm{v}}^{c}(j, t)$ and $\bigvee_{\mathrm{v} \in R\left(m_{j, t}\right)} B_{\mathrm{v}}^{\prime}(j, t) \vee$ $C_{\mathbf{v}}^{\prime c^{\prime}}(j, t)$ are $(\varepsilon(t), 1 / t)$-rigidly apart. Then for any $\delta<\varepsilon(t) / 2$ and any $\delta$ match of these two, for all but $t^{-1 / 4}$ of the $F_{r}$, for all but $t^{-1 / 4}$ of the $S(r, \sigma)$, for all but $t^{-1 / 4}$ of the atoms $E \in \bigvee_{\mathscr{M}(r)} \bar{B}_{\mathbf{v}}(j, t) \vee \bar{C}_{\mathbf{v}}^{c}(j, t) \vee \bar{B}_{\mathbf{v}}^{\prime}(j, t) \vee \bar{C}_{\mathbf{v}}^{\prime c^{\prime}}(j, t)$, $\bigvee_{\mathrm{v} \in S(r, \sigma)} \bar{B}_{\mathrm{v}}(j, t) \vee \bar{C}_{\mathrm{v}}^{c}(j, t) / F_{r} \cap E$ and $\bigvee_{\mathrm{v} \in S(r, \sigma)} \bar{B}_{\mathrm{v}}^{\prime}(j, t) \vee \bar{C}_{\mathrm{v}}^{\prime c^{\prime}}(j, t) / F_{r} \cap E$ are still $\left(\varepsilon(t) / 2,(k !)^{2} t^{-1 / 4}\right)$-rigidly apart.

Proof. If the lemma is false then there exists some $\delta<\varepsilon(t) / 2$ and a $\delta$ joining $\vee_{\mathrm{v} \in R\left(m_{j, t}\right)} \bar{B}_{\mathrm{v}} \vee \bar{C}_{\mathrm{v}}^{c} \vee \bar{B}_{\mathrm{v}}^{\prime} \vee \bar{C}_{\mathrm{v}}^{\prime c^{\prime}}$ such that for more than $t^{-1 / 4}$ of the $F_{r}, \exists S(r, \sigma)$ with $|S(r, \sigma)|>t^{-1 / 4}\left(2 m_{j, t}+1\right)^{n}(k !)^{-2}$, such that for more than $t^{-1 / 4}$ of the atoms $E \in \bigvee_{\mathrm{v} \in S(r, \sigma)^{c}} \bar{B}_{\mathrm{v}} \vee \bar{C}_{\mathrm{v}}^{c} \vee \bar{B}_{\mathrm{v}}^{\prime} \vee \bar{C}_{\mathrm{v}}^{\prime c^{\prime}}$, there exists a joining $\bigvee_{\mathbf{v} \in S(r, \sigma)} \widetilde{B}_{\mathbf{v}} \vee \widetilde{C}_{\mathbf{v}}^{c} \vee \widetilde{B}_{\mathbf{v}}^{\prime} \vee \widetilde{C}_{\mathbf{v}}^{\prime c^{\prime}}(j, t, r, E)$ of $\bigvee_{\mathbf{v} \in S(r, \sigma)} \bar{B}_{\mathbf{v}}(j, t) \vee \bar{C}_{\mathbf{v}}^{c}(j, t) / F_{r} \cap E$ and $\left.\bigvee_{\mathrm{v} \in S(r, \sigma)} \bar{B}_{\mathrm{v}}^{\prime}(j, t) \vee \bar{C}_{\mathrm{v}}^{\prime c^{\prime}}(j, t) / F\right) r \cap E$ which satisfies both 


$$
d_{|S(r, \sigma)|}\left(\bigvee_{\mathbf{v} \in S(r, \sigma)} \widetilde{B}_{\mathbf{v}}(j, t, r, E), \bigvee_{\mathbf{v} \in S(r, \sigma)} \widetilde{B}_{\mathbf{v}}^{\prime}(j, t, r, E)\right)<\frac{\varepsilon(t)}{2}
$$

and

$$
d_{|S(r, \sigma)|}\left(\bigvee_{\mathbf{v} \in S(r, \sigma)} \tilde{C}_{\mathbf{v}}^{c}(j, t, r, E), \bigvee_{\mathbf{v} \in S(r, \sigma)} \widetilde{C}_{\mathbf{v}}^{\prime c^{\prime}}(j, t, r, E)\right)<1-(k !)^{2} t^{-1 / 4} .
$$

Use the argument of Lemma 1 to see that this implies there must exist an $\varepsilon(t)$-joining $\bigvee_{\mathbf{v} \in R\left(m_{j, t}\right)} \widehat{B}_{\mathbf{v}} \vee \widehat{C}_{\mathbf{v}}^{c} \vee \widehat{B}_{\mathbf{v}}^{\prime} \vee \widehat{C}_{\mathbf{v}}^{\prime c^{\prime}}$ of the original arrays with

$$
\bar{d}_{(2 m(j, t)+1)^{n}}\left(\bigvee_{\mathbf{v} \in R\left(m_{j, t}\right)} \widehat{C}_{\mathbf{v}}^{c}, \bigvee_{\mathbf{v} \in R\left(m_{j, t}\right)} \widehat{C}_{\mathbf{v}}^{\prime c^{\prime}}\right)<1-t^{-3 / 4}\left(1-t^{-1 / 4}\right)<1-t^{-1},
$$

for $t>16(k !)^{-8}$. This is a contradiction, and thus the lemma must hold.

We can now prove the following lemma.

Lemma 4. In case (ii) of Lemma 2, we can construct a new array, disjointly colored in two ways, a new $\bar{\varepsilon}(t)>0$, so that the set of pairs $\hat{\mathscr{C}},(\bar{\varepsilon}(t), 1 / t)$ rigidly apart, is one larger than the set $\mathscr{C}$.

Proof. Suppose we are in case (ii) of Lemma 2, $\left(c, c^{\prime}\right) \notin \mathscr{C}$. Given any $A$, choose $t \in T(A)$. There exists $\hat{\varepsilon}(t, A)>0$ such that for any $\delta>0$, we can choose arbitrarily large $j$ and a $\delta$-match such that there exists $r, \sigma$, and $E$ with $\bigvee_{\mathbf{v} \in S(r, \sigma)} \bar{B}_{\mathbf{v}}(j, t) \vee \bar{C}_{\mathbf{v}}^{c}(j, t) / F_{r} \cap E$ and $\bigvee_{\mathbf{v} \in S(r, \sigma)} \bar{B}_{\mathbf{v}}^{\prime}(j, t) \vee \bar{C}_{\mathbf{v}}^{\prime c^{\prime}}(j, t) / F_{r} \cap$ $E, \quad(\hat{\varepsilon}(t, A), A)$-rigidly apart.

Let $\left(\bar{c}, \bar{c}^{\prime}\right) \in \mathscr{C}$. Use Lemma 3 to choose the same $r, \sigma, E$ so that

$$
\bigvee_{\mathbf{v} \in S(r, \sigma)} \bar{B}_{\mathbf{v}}(j, t) \vee \bar{C}_{\mathbf{v}}^{\bar{c}}(j, t) / F_{r} \cap E
$$

and $\bigvee_{\mathbf{v} \in S(r, \sigma)} \bar{B}_{\mathbf{v}}^{\prime}(j, t) \vee \bar{C}_{\mathbf{v}}^{\prime \bar{c}^{\prime}}(j, t) / F_{r} \cap E$ are $\left(\varepsilon(t) / 2,(k !)^{2} t^{-1 / 4}\right)$-rigidly apart. Fix $t$ and $A$. Let $t^{\prime}=\min \left\{1 / A, t^{1 / 4}(k !)^{2}\right\}$ and $\bar{\varepsilon}\left(t^{\prime}\right)=\min \{\hat{\varepsilon}(t, A), \varepsilon(t) / 2\}$. Then for any $\left(\bar{c}, \bar{c}^{\prime}\right) \in \mathscr{C} \cup\left\{\left(c, c^{\prime}\right)\right\}$, these two colorings are $\left(\bar{\varepsilon}\left(t^{\prime}\right), 1 / t^{\prime}\right)$-rigidly apart, no matter how small $\delta$ is. Apply Lemmas 2.2 and 2.5 of [1] to see that $\delta$ can be chosen arbitrarily small, and $F_{r}, S(r, \sigma)$ and $E$ can still be chosen so that for fixed $t^{\prime}$, the uncolored distribution may be made arbitrarily close in $\bar{d}$ to $\bigvee_{\mathrm{v} \in S(r, \sigma)} T_{\mathrm{v}} P$. This proves the lemma.

We have constructed a single uncolored array, but would now like to see that there is a single disjoint coloring from which the rigidly apart pairs are chosen. We need the following lemma, which is proved just as Lemma 3.

Lemma 5. If $\bigvee_{\mathbf{v} \in R(m)} B_{\mathbf{v}} \vee C_{\mathbf{v}}$ and $\bigvee_{\mathbf{v} \in R(m)} B_{\mathbf{v}} \vee C_{\mathbf{v}}^{\prime}$ are two colored distributions which are $(\varepsilon, A)$-rigidly apart, then for any partition $\left\{F_{r}\right\}$, for all but $A^{1 / 2}$ of the $F_{r}, \bigvee_{\mathrm{v} \in R(m)} B_{\mathrm{v}} \vee C_{\mathrm{v}} / F_{r}$ and $\bigvee_{\mathrm{v} \in R(m)} B_{\mathrm{v}} \vee C_{\mathrm{v}}^{\prime} / F_{r}$ are still $\left(\varepsilon, A^{1 / 2}\right)$-rigidly apart. 
Suppose now that a single distribution is disjointly colored in two different ways. Let $F_{r}$ be the partition of $X$ into at most $(k !)^{2}$ sets, on each of which, one disjoint coloring is the same as the other, except perhaps in a different order. Thus on each $F_{r}$, there exists a bijective function $\phi_{r}$ which reindexes the colorings. Specifically, suppose for two disjointly colored arrays, there exists an $\varepsilon(t)>0$ and $\left(c, c^{\prime}\right) \in \mathscr{C}$ so that $\bigvee_{\mathbf{v} \in R\left(m_{j, t}\right)} B_{\mathbf{v}}(j, t) \vee$ $C_{\mathbf{v}}^{c}(j, t)$ and $\bigvee_{\mathbf{v} \in R\left(m_{j, t}\right)} B_{\mathbf{v}}^{\prime}(j, t) \vee C_{\mathbf{v}}^{\prime c^{\prime}}(j, t)$ are $(\varepsilon(t), 1 / t)$-rigidly apart. By Lemma 5 , for all but $T^{-1 / 2}$ of the $F_{r}, \bigvee_{\mathrm{v} \in R\left(m_{j, t}\right)} B_{\mathrm{v}}(j, t) \vee C_{\mathrm{v}}^{c}(j, t) / F_{r}$ and $\bigvee_{\mathbf{v} \in R\left(m_{j, t}\right)} B_{\mathbf{v}}^{\prime}(j, t) \vee C_{\mathbf{v}}^{\prime c^{\prime}}(j, t) / F_{r}$ are $\left(\varepsilon(t), t^{-1 / 2}\right)$-rigidly apart. Reindex to see that $\bigvee_{\mathrm{v} \in R\left(m_{j, t}\right)} B_{\mathbf{v}}(j, t) \vee C_{\mathbf{v}}^{c}(j, t) / F_{r}$ and $\bigvee_{\mathbf{v} \in R\left(m_{j, t}\right)} B_{\mathbf{v}}^{\prime}(j, t) \vee C_{\mathbf{v}}^{\phi_{r}\left(c^{\prime}\right)}(j, t) / F_{r}$, from a single colored array, are $\left(\varepsilon(t), t^{-1 / 2}\right)$-rigidly apart. Use this to prove the following corollary.

Corollary 6. In case (ii) of Lemma 2, we can construct a single disjointly colored array, and a set of pairs $\mathscr{C}^{\prime}$ with $\left|\mathscr{C}^{\prime}\right|=|\mathscr{C}|+1$, such that any pair of colorings in $\mathscr{C}^{\prime}$, from this single array, are $\left(\varepsilon^{\prime}(t), 1 / t\right)$-rigidly apart.

Using this construction, we can now build a single disjointly colored array, a function $\varepsilon(t)>0$, and a maximal set $\mathscr{C}$ of pairs which are $(\varepsilon(t), 1 / t)$-rigidly apart, such that for any $\left(c, c^{\prime}\right) \notin \mathscr{C}$, for any $\varepsilon_{1}>0, \exists T$ such that for all $\varepsilon_{2}>0, \exists J$ such that for $t \geq T, j \geq J$, the distributions $\bigvee_{\mathrm{v} \in R\left(m_{j, t}\right)} B_{\mathrm{v}}(j, t) \vee$ $C_{\mathbf{v}}^{c}(j, t)$ and $\bigvee_{\mathbf{v} \in R\left(m_{j, t}\right)} B_{\mathbf{v}}(j, t) \vee C_{\mathbf{v}}^{c^{\prime}}(j, t)$ are $\left(\varepsilon_{2}, \varepsilon_{1}\right)$-close. We call such a maximal arrangement.

Theorem 7. If in any maximal arrangement, $\operatorname{card}(\mathscr{C})=0$, then $\left(\mathscr{T}^{g}, \bar{P}\right)$ is Bernoulli.

Proof. Since $\operatorname{card}(\mathscr{C})=0$, for all $\left(c, c^{\prime}\right)$, for all $\varepsilon_{1}, \varepsilon_{2}$, and large $j, t$,

$\bar{d}_{(2 m(j, t)+1)^{n}}\left(\bigvee_{\mathbf{v} \in R\left(m_{j, t}\right)} B_{\mathbf{v}}(j, t) \vee C_{\mathbf{v}}^{c}(j, t), \bigvee_{\mathbf{v} \in R\left(m_{j, t}\right)} B_{\mathbf{v}}(j, t) \vee C_{\mathbf{v}}^{c^{\prime}}(j, t)\right)<\varepsilon_{1}+\varepsilon_{2}$

Use this, together with the fact that $(\mathscr{T}, P)$ is extremal (since finitely determined), via the proof of Theorem 2.7 in [1], to see that $\left(\mathscr{T}^{g}, \bar{P}\right)$ is extremal and hence Bernoulli.

Now we show that if $\mathscr{C} \neq \varnothing$ then $\left(\mathscr{T}^{g}, \bar{P}\right)$ is not Bernoulli.

We will first select a maximal arrangement with $\bigvee_{\mathbf{v} \in R\left(m_{j, t}\right)} B_{\mathbf{v}}(j, t)$ equal to $\bigvee_{\mathbf{v} \in R\left(m_{j, t}\right)} T_{\mathbf{v}} P$. In a maximal arrangement, the set $\{1,2, \ldots, k\}$ breaks into equivalence classes $M_{1}, \ldots, M_{s}$, such that $\left(c, c^{\prime}\right) \in \mathscr{C}$ if and only if $c$ and $c^{\prime}$ belong to different $M_{i}$ 's. These $M_{i}$ 's will be used to construct a finite rotation factor for $\left(\mathscr{T}^{g}, \bar{P}\right)$.

The proof of the following technical lemma is essentially the same as the proof of Lemmas 3 and 5.

Lemma 8. If $\bigvee_{\mathrm{v} \in R(m)} B_{\mathrm{v}} \vee C_{\mathrm{v}}$ and $\bigvee_{\mathrm{v} \in R(m)} B_{\mathrm{v}} \vee C_{\mathrm{v}}^{\prime}$ are two colored distributions which are $(\varepsilon, A)$-rigidly apart, and $S \subset R(m)$, then for all but $A^{1 / 2}$ of the atoms $E$ in $\bigvee_{\mathrm{v} \in S^{c}} B_{\mathrm{v}} \vee C_{\mathrm{v}} \vee C_{\mathrm{v}}^{\prime}$, the distributions $\bigvee_{\mathrm{v} \in S} B_{\mathrm{v}} \vee C_{\mathrm{v}} / E$ and $\bigvee_{\mathrm{v} \in S} B_{\mathrm{v}} \vee C_{\mathrm{v}}^{\prime}$ are still $\left(\varepsilon, A^{1 / 2}(2 m+1)^{n} /|S|\right)$-rigidly apart. 
We use Lemma 8 to prove the following analog of Lemma 2.8 of [1].

Lemma 9. In a maximal arrangement, for any $\left(c, c^{\prime}\right) \notin \mathscr{C}$, and $\varepsilon_{1}>0, \alpha<1$, there is a $T$ so that for any $t>T$ and $\varepsilon_{2}>0$, there exist $J, \delta, m$, so that for $j>J$ and any $(\alpha, \delta, m)$-subset $S \subset R\left(m_{j, t}\right)$, we have both

(i) for all but $\varepsilon_{2}$ of the atoms $E \in \bigvee_{\mathbf{v} \in S^{c}} B_{\mathbf{v}}(j, t) \vee \bigvee_{c=1}^{k} C_{\mathbf{v}}^{c}(j, t)$,

$$
\bar{d}_{\operatorname{card}(S)}\left(\bigvee_{\mathbf{v} \in S} B_{\mathbf{v}}(j, t) / E, \bigvee_{\mathbf{v} \in S} B_{\mathbf{v}}(j, t)\right)<\varepsilon_{2},
$$

and

(ii) for all but $2 t^{-1 / 2}$ of the atoms $E, \bigvee_{\mathbf{v} \in S} B_{\mathbf{v}}(j, t) \vee C_{\mathbf{v}}^{c}(j, t) / E$ and

$$
\bigvee_{\mathbf{v} \in S} B_{\mathbf{v}}(j, t) \vee C_{\mathbf{v}}^{c^{\prime}}(j, t)
$$

are $\left(\varepsilon_{2}, \varepsilon_{1}\right)$-close.

Proof. Prove (i) just as in Lemma 2.8 of [1]. To prove (ii), we will assume it is false and show that this contradicts the maximality of $\mathscr{C}$.

Suppose (ii) is false. Then for some $\left(c, c^{\prime}\right) \notin \mathscr{C}$, there exists $\varepsilon_{1}>0$, $\alpha<1$, infinitely many $t$ and $\varepsilon_{2}>0$, so that for infinitely many $j$ and for all $\delta, m$, there exists an $(\alpha, \delta, m)$-subset $S_{j} \subset R\left(m_{j, t}\right)$ such that for more than $2 t^{-1 / 2}$ of the atoms $E$, we have that $\bigvee_{\mathrm{v} \in S} B_{\mathrm{v}}(j, t) \vee C_{\mathrm{v}}^{c}(j, t) / E$ and $\bigvee_{\mathbf{v} \in S} B_{\mathbf{v}}(j, t) \vee C_{\mathbf{v}}^{c^{\prime}}(j, t)$ are not $\left(\varepsilon_{2}, \varepsilon_{1}\right)$-close. In particular, they must be $\left(\varepsilon_{2}, 1-\varepsilon_{1}\right)$-rigidly apart.

Let $\left(\bar{c}, \bar{c}^{\prime}\right) \in \mathscr{C}$, so that $\bigvee_{\mathrm{v} \in R(m(j, t))} B_{\mathbf{v}}(j, t) \vee C_{\mathbf{v}}^{\bar{c}}(j, t)$ and $\bigvee_{\mathbf{v} \in R\left(m_{j, t}\right)} B_{\mathbf{v}}(j, t)$ $\vee C_{\mathbf{v}}^{\bar{c}^{\prime}}(j, t)$ are $(\varepsilon(t), 1 / t)$-rigidly apart. By Lemma 8 , for all but $t^{-1 / 2}$ of the atoms $E$ in $\bigvee_{\mathrm{v} \in S_{j}^{c}} B_{\mathrm{v}}(j, t) \vee C_{\mathrm{v}}^{\bar{c}}(j, t) \vee C_{\mathrm{v}}^{\bar{c}^{\prime}}(j, t)$ we have that $\bigvee_{\mathrm{v} \in S_{j}} B_{\mathbf{v}}(j, t) \vee$ $C_{\mathbf{v}}^{\bar{c}}(j, t) / E$ and $\bigvee_{\mathbf{v} \in S_{j}} B_{\mathbf{v}}(j, t) \vee C_{\mathbf{v}}^{\bar{c}^{\prime}}(j, t)$ are still $\left(\varepsilon(t), t^{-1 / 2}(2 m+1)^{n} /\left|S_{j}\right|\right)-$ rigidly apart.

Let $t^{\prime}=\min \left\{1 / \varepsilon_{1}, t^{1 / 2} \alpha\right\}$ and let $\varepsilon\left(t^{\prime}\right)=\min \left\{t^{-1 / 2}, \varepsilon(t), \varepsilon_{2}\right\}$. It can be shown that the pairs $\mathscr{C} \cup\left\{\left(c, c^{\prime}\right)\right\}$ are $\left(\varepsilon\left(t^{\prime}\right), 1 / t^{\prime}\right)$-rigidly apart, which contradicts the maximality of $\mathscr{C}$.

The following lemma is proven in essentially the same way as Lemma 9.

Lemma 10. If we have a maximal arrangement, then for any $\varepsilon_{1}$, there is a $T$ so that for $t>T$ and any $\varepsilon_{2}$, there is $a \delta$ and $a J$, so that for $j>J$ and any partition $\left\{F_{r}\right\}$ with more than $\delta$ of its mass in sets larger than $2^{-\delta(2 m(j, t)+1)^{n}}$, both

(i) for all but $\varepsilon_{2}$ of the $F_{r}$,

$$
\bar{d}_{\left(2 m_{j, l}+1\right)^{n}}\left(\bigvee_{\mathbf{v} \in R\left(m_{j, l}\right)} B_{\mathbf{v}}(j, t) / F_{r}, \bigvee_{\mathbf{v} \in R\left(m_{j, t}\right)} B_{\mathbf{v}}(j, t)\right)<\varepsilon_{2}
$$

and

(ii) for all but $2 t^{-1 / 2}$ of the $F_{r}, \bigvee_{\mathbf{v} \in R\left(m_{j, t}\right)} B_{\mathbf{v}}(j, t) \vee C_{\mathbf{v}}^{c}(j, t) / F_{r}$ and 


$$
\bigvee_{\mathbf{v} \in R\left(m_{j, t}\right)} B_{\mathbf{v}}(j, t) \vee C_{\mathbf{v}}^{c^{\prime}}(j, t)
$$

are $\left(\varepsilon_{2}, \varepsilon_{1}\right)$-close, for all $\left(c, c^{\prime}\right) \notin \mathscr{C}$.

Let $\mathscr{W}(j, t, m)$ be the set of vectors in $R\left(m_{j, t}-m\right)$ which are also in the integer span of

$$
\left\{\left(2\left[\frac{m_{j, t}}{m}\right]+1,0, \ldots, 0\right), \ldots,\left(0, \ldots, 0,2\left[\frac{m_{j, t}}{m}\right]+1\right)\right\},
$$

where [.] is the greatest integer function. The vectors in $\mathscr{W}(j, t, m)$ are those vectors which are the centers of grid cubes of size $(2 m+1)^{n}$ within the larger $R\left(m_{j, t}\right)$.

Lemma 11. In a maximal arrangement, for any $A>0$, there is $a T$, so that for $t>T$, and any $\varepsilon^{\prime}>0$, there are $M$ and $J$ so that for $m>M, j>J$, $\varepsilon^{\prime} m_{j, t}>m$, for all but a fraction $A$ of the values $\mathbf{w} \in \mathscr{W}(j, t, m)$, if $\left(c, c^{\prime}\right) \in$ $\mathscr{C}$ then $\bigvee_{\mathrm{v} \in R(\mathrm{w}, m)} B_{\mathrm{v}}(j, t) \vee C_{\mathrm{v}}^{c}(j, t)$ and $\bigvee_{\mathrm{v} \in R(\mathrm{w}, m)} B_{\mathrm{v}}(j, t) \vee C_{\mathrm{v}}^{c^{\prime}}(j, t)$ are $\left(\varepsilon(t)-2 \varepsilon^{\prime}, A\right)$-rigidly apart.

Proof. The proof of this lemma is analogous to that of Lemma 2.9 [1], using Lemma 9 in place of Lemma 2.8 of [1]. Also use the fact that $(c, c) \notin \mathscr{C}$ for any color $c$.

Finally, we see that we may work with the original process, instead of copies of it. The proof of the following lemma is analogous to that of Lemma 2.10 in [1].

Lemma 12. There is a maximal arrangement with

$$
\bigvee_{\mathbf{v} \in R\left(m_{j, t}\right)} B_{\mathbf{v}}(j, t)=\bigvee_{\mathbf{v} \in R\left(m_{j, t}\right)} T_{\mathbf{v}} P
$$

Call the above maximal arrangement a perfect arrangement. We must now show that this arrangement is, in some sense, unique.

Recall that $\{1,2, \ldots, k\}$ splits into subsets $M_{1}, \ldots, M_{s}$, so that $\left(c, c^{\prime}\right) \in$ $\mathscr{C}$ if and only if $c$ and $c^{\prime}$ belong to different sets $M_{i}$. Define the coloring $\bigvee_{\mathrm{v} \in R\left(m_{j, t}\right)} \bar{C}_{\mathrm{v}}^{i}(j, t)$ to be the coloring which gives each name in $\bigvee_{\mathrm{v} \in R\left(m_{j, t}\right)} T_{\mathrm{v}} P$ all the colorings it has from any term in $M_{i}$, each with equal probability. In effect, we are lumping together those colorings which are rigidly close to each other. We show that this coloring is essentially unique, as stated in the following lemma.

Lemma 13. Let $\bigvee_{\mathbf{v} \in R\left(m_{j, t}\right)} T_{\mathrm{v}} P \vee \bigvee_{c=1}^{k} C_{\mathrm{v}}^{c}(j, t), \mathscr{C}, \varepsilon(t)$ and $\bigvee_{\mathrm{v} \in R\left(m_{j, t}\right)} T_{\mathrm{v}} P \vee$ $\bigvee_{c=1}^{k} C_{\mathrm{v}}^{\prime c}(j, t), \mathscr{C}^{\prime}, \varepsilon^{\prime}(t)$ be two perfect arrangements. For any $\varepsilon>0$ there exists a $T$ so that for $t>T$, there exists a $J(t, \varepsilon)$ so that for $j>J(t, \varepsilon)$, for each $i$, there is an $i^{\prime}$ such that for all but $\varepsilon$ of the atoms $E$ in $\bigvee_{\mathrm{v} \in R\left(m_{j, t}\right)} T_{\mathrm{v}} P$,

$$
\bar{d}_{\left(2 m_{j, t}+1\right)^{n}}\left(\bigvee_{\mathrm{v} \in R\left(m_{j, t}\right)} \bar{C}_{\mathrm{v}}^{i}(j, t) / E, \bigvee_{\mathrm{v} \in R\left(m_{j, t}\right)} \bar{C}_{\mathrm{v}}^{\prime i^{\prime}}(j, t) / E\right)<\varepsilon
$$

Proof. If the lemma is false, there exists some $\varepsilon_{0}>0$ and infinitely many $t$ such that for each $t$, there exists infinitely many $j$ such that for each $i$, for 
more than $\varepsilon_{0}$ of the atoms $E$, the $\bar{d}$-distance above is larger than $\varepsilon_{0}$, for all $i^{\prime}$. Thus there exists $\left(c, c^{\prime}\right) \in \mathscr{C}^{\prime}$ and $\left(\bar{c}, \bar{c}^{\prime}\right) \notin \mathscr{C}$, and disjoint sets $F_{1}, F_{2}$ in $\bigvee_{\mathrm{v} \in R\left(m_{j, t}\right)} T_{\mathrm{v}} P$ with $\mu\left(F_{1}\right)=\mu\left(F_{2}\right)>\varepsilon_{0} / k^{4}$, such that

$$
\bigvee_{\mathbf{v} \in R\left(m_{j, t}\right)} T_{\mathbf{v}} P \vee C_{\mathbf{v}}^{\bar{c}}(j, t) / F_{1}=\bigvee_{\mathbf{v} \in R\left(m_{j, t}\right)} T_{\mathbf{v}} P \vee C_{\mathbf{v}}^{\prime c}(j, t) / F_{2}
$$

and

$$
\bigvee_{\mathbf{v} \in R\left(m_{j, t}\right)} T_{\mathbf{v}} P \vee C_{\mathbf{v}}^{\bar{c}^{\prime}}(j, t) / F_{1}=\bigvee_{\mathbf{v} \in R\left(m_{j, t}\right)} T_{\mathbf{v}} P \vee C_{\mathbf{v}}^{\prime^{c^{\prime}}}(j, t) / F_{2}
$$

for infinitely many $j$.

But in the above equalities, the left-hand sides are $\left(\varepsilon_{2}, \varepsilon_{1}\right)$-close, by Lemma 10 , as $\varepsilon_{2} \rightarrow 0$ in $j$, and $\varepsilon_{1} \rightarrow 0$ in $t$. Furthermore, the right-hand sides are still $\left(\varepsilon^{\prime}(t), t^{-1 / 2}\right)$-rigidly apart, by Lemma 5 . This is a conflict, and so the lemma is proven.

The following rigidity lemma is a consequence of Lemma 11.

Lemma 14. In a perfect arrangement, $\forall A>0, \exists T$ such that for $t>T$ and any $\varepsilon^{\prime}>0, \exists M$ and $J$ such that for $m>M, j>J, \varepsilon^{\prime} m_{j, t}>m$, for all but $A$ of the values $\mathbf{w} \in \mathscr{W}(j, t, m), \forall i, i^{\prime}, \bigvee_{\mathbf{v} \in R(\mathbf{w}, m)} T_{\mathbf{v}} P \vee \bar{C}_{\mathbf{v}}^{i}(j, t)$ and $\bigvee_{\mathbf{v} \in R(\mathbf{w}, m)} T_{\mathbf{v}} P \vee \bar{C}_{\mathbf{v}}^{i^{\prime}}(j, t)$ are $\left(\varepsilon(t)-2 \varepsilon^{\prime}, A\right)$-rigidly apart.

Lemma 14 says that the colored process may be broken into rigid grid-pieces. Generalize this further to see that any "appropriate piece" of the colored process is rigid. The proof of Lemma 15 is analogous to that of Lemma 2.16 of [1].

Lemma 15. In a perfect arrangement, $\forall A>0, \exists T$ such that for $t>T$ and any $\varepsilon^{\prime}>0, \exists M$ and $J$ such that for $m>M, j>J, \varepsilon^{\prime} m_{j, t}>m$, for all but $A$ of the values $\mathbf{w} \in R\left(m_{j, t}\right), \forall i, i^{\prime}, \bigvee_{\mathbf{v} \in R(\mathbf{w}, m)} T_{\mathbf{v}} P \vee \bar{C}_{\mathbf{v}}^{i}(j, t)$ and $\bigvee_{\mathbf{v} \in R(\mathbf{w}, m)} T_{\mathbf{v}} P \vee \bar{C}_{\mathbf{v}}^{i^{\prime}}(j, t)$ are $\left(\varepsilon(t)-2 \varepsilon^{\prime}, A\right)$-rigidly apart.

Corollary 16. In a perfect arrangement, $\forall \varepsilon>0, \exists T$ such that for $t>T, \exists M$ and $J$ such that for $m>M, j>J$, for all but $\varepsilon$ of the $\mathbf{w} \in R\left(m_{j, t}-m\right)$, for every $i$ there exists a unique $i^{\prime}$ such that

$$
\bar{d}_{(2 m+1)^{n}}\left(\bigvee_{\mathbf{v} \in R(m)} \bar{C}_{\mathbf{v}}^{i}(m), \bigvee_{\mathbf{v} \in R(m)} T_{\mathbf{w}}^{g}\left(\bar{C}_{\mathbf{v}-\mathbf{w}}^{i^{\prime}}\left(m_{j, t}\right)\right)\right)<\varepsilon .
$$

Proof. This is a consequence of Lemma 15, and the uniqueness described in Lemma 13.

For each $m_{j, t}$, define a partition $\left\{H_{1}\left(m_{j, t}\right), \ldots, H_{s}\left(m_{j, t}\right)\right\}$ of $\widehat{X}$ where $\hat{x} \in H_{i}\left(m_{j, t}\right)$ if and only if the $\mathscr{T}^{g}-\bar{P}-\left(2 m_{j, t}+1\right)^{n}$ name of $\hat{x}$ is a name in $\bigvee_{\mathrm{v} \in R\left(m_{j, t}\right)} \bar{C}_{\mathrm{v}}^{i}\left(m_{j, t}\right)$.

Corollary 17. For every $\varepsilon>0, \exists T$ such that for $t>T, \exists M$ and $J$ such that for $m>M, j>J$, for all but $\varepsilon$ of the $\mathbf{w} \in R\left(m_{j, t}-m\right)$, for every $i$, there exists a unique $r$ such that $\hat{\mu}\left(H_{i}(m) \cap T_{\mathbf{w}}^{g}\left(H_{r}\left(m_{j, t}\right)\right)\right)>(1-\varepsilon) \hat{\mu}\left(H_{i}(m)\right)$.

Proof. Any name $\eta_{m}^{i}$ in $H_{i}(m)$, by Corollary 16, must occur mostly in exactly one $T_{\mathrm{w}}^{g}\left(H_{r}\left(m_{j, t}\right)\right)$. Specifically, for all but $\varepsilon$ of the $\mathbf{w} \in R\left(m_{j, t}-m\right)$, for 
every $i$, there exists a unique $r$, such that for all but $\varepsilon$ of the $\eta_{m}^{i}$ in $H_{i}(m)$, $\hat{\mu}\left(\eta_{m}^{i} \cap T_{\mathrm{w}}^{g}\left(H_{r}\left(m_{j, t}\right)\right)\right)>(1-\varepsilon) \hat{\mu}\left(\eta_{m}^{i}\right)$. Hence $\hat{\mu}\left(H_{i}(m) \cap T_{\mathrm{w}}^{g}\left(H_{r}\left(m_{j, t}\right)\right)\right)>$ $(1-\varepsilon) \hat{\mu}\left(H_{i}(m)\right)$.

Apply Corollary 17 using the method displayed in the proof of Theorem 2.19 [1], to show that for any $\varepsilon>0$, there exists a set $A$ of measure larger than $(1-\varepsilon) / k$ such that $\hat{\mu}\left(T_{\mathbf{v}}^{g}(A) \cap A\right)>\hat{\mu}(A)-\varepsilon$, for a set of $\mathbf{v} \in \mathbb{Z}^{n}$ of full density. We conclude that such an extension is not weakly mixing. Specifically, this completes the proof of the following theorem.

Theorem 18. If in any maximal arrangement, $\operatorname{card}(\mathscr{C}) \neq 0$, then $\left(\mathscr{T}^{g}, \bar{P}\right)$ is not weakly mixing.

This concludes the proof of the classification theorem. We now apply the result to carry out the classification.

Let $(X, \mathscr{A}, \mu), \mathscr{T}$ be a $\mathbb{Z}^{n}$-Bernoulli shift and let $(\widehat{X}, \widehat{\mathscr{A}}, \hat{\mu}), \mathscr{T} g$ be a $k$-point extension of $\mathscr{T}$, given by the cocycle $\mathbf{g}=\left(g_{1}, \ldots, g_{n}\right)$. Define the full extension $\widetilde{\mathscr{T}^{g}}$ of $\mathscr{T}^{g}$ by $\widetilde{\mathscr{T}^{g}}=\left\{\widetilde{\mathscr{T}}_{\mathbf{e}_{i}}^{g}\right\}, \widetilde{T}_{\mathbf{e}_{i}}^{g}: X \times S_{k} \rightarrow X \times S_{k}, \widetilde{T}_{\mathbf{e}_{i}}^{g}(x, \sigma)=$ $\left(T_{\mathbf{e}_{i}} x, g_{i}(x) \circ \sigma\right)$. View this full extension as a $k$ !-point extension of the base Bernoulli shift. We may consider this full extension $\widetilde{\mathscr{T}}^{g}$ as a part of a larger $\mathbb{Z}^{n} \times S_{k}$-action. In particular, for any $\gamma \in S_{k}$, let $\widetilde{T}_{\gamma}(x, \sigma)=(x, \sigma \circ \gamma)$. For each $\mathbf{v} \in \mathbb{Z}^{n}$ and $\gamma \in S_{k}$, let $U_{\mathbf{v}, \gamma}^{g}=\widetilde{T}_{\mathbf{v}}^{g} \circ \widetilde{T}_{\gamma}=\widetilde{T}_{\gamma} \circ \widetilde{T}_{\mathbf{v}}^{g}$, so that $\mathscr{U}^{g}=\left\{U_{\mathbf{v}, \gamma}^{g}\right\}_{(\mathbf{v}, \gamma) \in \mathbb{Z}^{n} \times S_{k}}$ is a $\mathbb{Z}^{n} \times S_{k}$-action on $X \times S_{k}$.

It is easy to show [4] that any two $k$-point extensions $\mathscr{T}^{g}$ and $\mathscr{T}^{g^{\prime}}$ are isomorphic, by an isomorphism which respects their common Bernoulli factor, if and only if their corresponding $\mathbb{Z}^{n} \times S_{k}$-actions $\mathscr{U}^{g}$ and $\mathscr{U}^{g^{\prime}}$ are themselves isomorphic. We thus concentrate on classifying these $\mathbb{Z}^{n} \times S_{k}$-actions.

Suppose that the full extension $\widetilde{\mathscr{T}^{g}}$ is not $\mathbb{Z}^{n}$-Bernoulli. This implies, by the Classification Theorem, that $\widetilde{\mathscr{T} g}$ is not weakly mixing. Let $\pi(\widetilde{\mathscr{T} g})$ be the Pinkser algebra of $\widetilde{\mathscr{T}^{g}}$. For any $\gamma \in S_{k}, \widetilde{T}_{\gamma}\left(\pi\left(\widetilde{\mathscr{T}}^{g}\right)\right)=\pi(\widetilde{\mathscr{T}} g)$. The process $\left.\widetilde{\mathscr{T}^{g}}\right|_{\pi(\widetilde{\mathscr{T} g})}$ is a zero-entropy process, while $\left.\widetilde{\mathscr{T}}^{g}\right|_{\mathscr{A} \times\left\{S_{k}, \varnothing\right\}}$ is a $\mathbb{Z}^{n}$-Bernoulli process. Thus $\pi\left(\widetilde{\mathscr{T}^{g}}\right) \perp \mathscr{A} \times\left\{S_{k}, \varnothing\right\}$. Specifically, $\pi\left(\widetilde{\mathscr{T}^{g}}\right)$ is a finite algebra consisting of at most $k$ ! sets, and $\left\{\left.\widetilde{\mathscr{T}_{\gamma}}\right|_{\pi(\widetilde{\mathscr{T} g})}\right\}_{\gamma \in S_{k}}$ is an ergodic action of $S_{k}$ on this finite algebra. Such an action is isomorphic to right multiplication on the right cosets of some closed subgroup $H \subset S_{k}$. In this case, for example, a choice for $H$ is the subgroup of $S_{k}$ which fixes a particular atom of $\pi\left(\overline{\mathscr{T}}^{g}\right)$.

Let $P: X \times S_{k} \rightarrow H \backslash S_{k}$ be projection onto this factor, $P(x, \sigma)=H \sigma$. For almost every $x \in X, P^{-1}(H) \cap\left(x \times S_{k}\right)=x \times\left(\gamma_{x} H\right)$, where $\gamma_{x} H$ is some left coset of $H$ in the fiber over $x$. This $\gamma_{x}$ can be chosen to be a measurable function of $x$.

Consider a new $\mathbb{Z}^{n} \times S_{k}$-action $\overline{\mathscr{U}}^{g}=\left\{\overline{\mathscr{U}}_{\mathrm{v}, \gamma}^{g}\right\}=\left\{\bar{T}_{\mathrm{v}}^{g} \circ \bar{T}_{\gamma}\right\}$, isomorphic to $\mathscr{U}^{g}$ where $\bar{U}_{\mathbf{v}, \gamma}^{g}=\phi U_{\mathbf{v}, \gamma}^{g} \phi^{-1}, \phi=\left(\mathrm{id}, \gamma_{x}^{-1}\right)$. Specifically, $\bar{U}_{\mathbf{v}, \gamma}^{g}(x, \sigma)=$ $\left(T_{\mathrm{v}} x, \bar{g}_{\mathrm{v}}(x) \circ \sigma \circ \gamma\right)$ where $\bar{g}_{\mathrm{v}}(x)=\gamma_{T_{\mathrm{v} x}}^{-1} \circ g_{\mathrm{v}}(x) \circ \gamma_{x}$. The Pinsker algebra of $\overline{\mathscr{T}}^{g}$ satisfies $\pi\left(\overline{\mathscr{T}}^{g}\right)=\phi\left(\pi\left(\widetilde{\mathscr{T}}^{g}\right)\right)=\left\{X \times H A \mid A \subset S_{k}\right\}$. The atoms of $\pi\left(\overline{\mathscr{T}}^{g}\right)$ are precisely the $X \times H_{\gamma}$, so that $\bar{T}_{\mathbf{v}}^{g}(x \times H)=T_{\mathbf{v}} x \times H \gamma(\mathbf{v})$, for some $\gamma(\mathbf{v}) \in S_{k}$. But also $\bar{T}_{\mathbf{v}}^{g}(x \times H)=T_{\mathbf{v}} x \times \bar{g}_{\mathbf{v}}(x) H$, so that $H \gamma(\mathbf{v})=\bar{g}_{\mathbf{v}}(x) H$. Therefore 
$\bar{g}_{\mathbf{v}}(x)=h(x) \gamma(\mathbf{v})$ for some $h(x) \in H$. This implies $\gamma(\mathbf{v})^{-1} H \gamma(\mathbf{v})=H$, so that $\gamma(\mathbf{v}) \in N_{S_{k}}(H)$, the normalizer of $H$ in $S_{k}$. Specifically, $\left.\overline{\mathscr{T}}^{g}\right|_{\pi\left(\overline{\mathscr{T}}^{g}\right)}$ is isomorphic to right multiplication by $\{\gamma(\mathbf{v})\}_{\mathbf{v}}$ on the right cosets of $H$.

The choice of $H$ is unique up to inner automorphism. For a fixed $H$ and $\mathbf{v}$, the choice of $\gamma(\mathbf{v})$ is not unique, but the choice of $H \gamma(\mathbf{v})$ is unique. Thus, to any finite extension $\mathscr{T}^{g}$, we may assign a unique equivalence class $\mathscr{H}\left(\mathscr{T}^{g}\right)=\left\{(H, \gamma(\mathbf{v})) \mid H\right.$ is a closed subgroup of $\left.S_{k}, \gamma(\mathbf{v}) \in N_{S_{k}}(H), \mathbf{v} \in \mathbb{Z}^{n}\right\}$ where $(H, \gamma(\mathbf{v})) \sim\left(H^{\prime}, \gamma^{\prime}(\mathbf{v})\right)$ if and only if there exists some $\alpha \in S_{k}, h \in H$ such that $\alpha^{-1} H \alpha=H^{\prime}$ and $\alpha^{-1}(h \gamma(\mathbf{v})) \alpha=\gamma^{\prime}(\mathbf{v})$.

Lemma 19. Two k-point extensions $\mathscr{T}^{g}$ and $\mathscr{T}^{g^{\prime}}$ of a $\mathbb{Z}^{n}$-Bernoulli shift $\mathscr{T}$ satisfy $\mathscr{H}\left(\mathscr{T}^{g}\right)=\mathscr{H}\left(\mathscr{T}^{\prime}\right)$ if and only if they are isomorphic via an isomorphism which preserves the common Bernoulli factor.

Proof. For $\mathbf{v} \in \mathbb{Z}^{n}$, let $S_{\mathbf{v}}=\bar{T}_{\gamma(\mathbf{v})^{-1}} \bar{T}_{\mathbf{v} \mid X \times H}^{g}$. For $h \in H$, let $S_{h}=\bar{T}_{h_{\mid X \times H}}$. Let $S_{(\mathbf{v}, h)}=S_{\mathbf{v}} S_{h}=S_{\gamma(\mathbf{v})^{-1} h \gamma(\mathbf{v})} S_{\mathbf{v}}$, where $S_{\alpha \beta}(x, \sigma)=(x, \sigma \beta \alpha)$ for $\alpha, \beta \in H$, $(x, \sigma) \in X \times H$. It can be shown that

$$
S_{\left(\mathbf{v}_{1}, h_{1}\right)} S_{\left(\mathbf{v}_{2}, h_{2}\right)}(x, H)=S_{\left(\mathbf{v}_{1}+\mathbf{v}_{2}, h_{2} \varphi\left(\mathbf{v}_{2}\right)^{-1} h_{1} \gamma\left(\mathbf{v}_{2}\right)\right)}(x, H),
$$

which we write as $S_{\left(\mathbf{v}_{1}+\mathbf{v}_{2}, h_{2} \phi_{v_{2}}\left(h_{1}\right)\right)}(x, H)$, so that $\left\{S_{(\mathbf{v}, h)}\right\}_{\mathbf{v} \in \mathbb{Z}^{n}, h \in H}$ is a $\mathbb{Z}^{n} \otimes^{\phi} S_{k^{-}}$ action on $X \times H$, [6]. The isomorphism theorem for Bernoulli free $\mathbb{Z}$-skewcompact group actions [6] may be easily extended to Bernoulli free $\mathbb{Z}^{n}$-skewcompact group actions. This theorem applies if $\mathscr{S}=\left\{S_{\mathrm{v}}\right\}_{\mathrm{v} \in \mathbb{Z}^{n}}$ is Bernoulli.

The point is that $\mathscr{S}$ acting on the cosets $(X \times H)$ can be thought of as a finite extension of the original base Bernoulli shift, where "finite" corresponds to the number of cosets of $H$ in $S_{k}$. If $\mathscr{S}$ is weakly mixing then the classification theorem implies that it is $\mathbb{Z}^{n}$-Bernoulli. Note that $\pi\left(\left\{\bar{T}_{\gamma(\mathbf{v})^{-1}} \bar{T}_{\mathbf{v}}^{g}\right\}_{\mathbf{v} \in \mathbb{Z}^{n}}\right)=\pi\left(\overline{\mathscr{T}}^{g}\right)$ and that $X \times H$ is an atom of $\pi\left(\overline{\mathscr{T}}^{g}\right)$, so that $\mathscr{S}$ is weakly mixing, and hence Bernoulli.

Similarly, construct $\mathscr{S}^{\prime}$ to correspond to $\mathscr{T} g^{\prime}$. The $\mathbb{Z}^{n}$-skew-compact isomorphism theorem implies that such an $\mathscr{S}$ and $\mathscr{S}^{\prime}$ must be isomorphic. Use this isomorphism to construct an isomorphism between the full $\mathbb{Z}^{n} \times S_{k}$-actions, $\overline{\mathscr{U}}^{g}$ and $\overline{\mathscr{U}}^{g^{\prime}}$, [4]. As discussed earlier, this will give rise to an isomorphism between the $k$-point extensions $\mathscr{T}^{g}$ and $\mathscr{T}^{g^{\prime}}$ which preserves the common Bernoulli factor.

In summary, we have shown that the nonweakly mixing finite extensions of a $\mathbb{Z}^{n}$-Bernoulli shift may be completely classified by the constructed $\mathscr{H}\left(\mathscr{T}^{g}\right)$. These extensions have been classified up to factor isomorphism. If we do not require that this isomorphism preserve the common Bernoulli factor, then the nonweakly mixing finite extensions of a $\mathbb{Z}^{n}$-Bernoulli shift may be completely classified by the Pinsker algebra $\pi\left(\mathscr{T}^{g}\right)$. Specifically, if such an extension is ergodic then it is ergodic on the Pinsker algebra, and hence is cyclic. If the extension is nonergodic then the atoms of the Pinsker algebra may be divided into subsets (ergodic components) on each of which the extension is cyclic. Any two such extensions will be isomorphic if and only if their Pinsker algebras have the same number of ergodic components and these ergodic components may be matched by a bijection which preserves the number of Pinsker algebra atoms within each ergodic component. 
The classification theorem extends to $\mathbb{R}^{n}$-Bernoulli flows and generalized $\mathbb{Z}^{n}$ Bernoulli shifts, as defined in [1].

Corollary 20. The finite extension of a generalized $\mathbb{Z}^{n}$-Bernoulli shift either is itself a generalized $\mathbb{Z}^{n}$-Bernoulli shift or has a finite rotation factor.

The proof of Corollary 19 follows exactly that of Corollary 2.21 of [1].

Corollary 21. The finite extension of an $\mathbb{R}^{n}$-Bernoulli flow either is itself an $\mathbb{R}^{n}$ Bernoulli flow or is nonergodic.

Proof. Let $\mathscr{S}^{g}$ be a $k$-point extension of the $\mathbb{R}^{n}$-Bernoulli flow $\mathscr{S}$. Since $\mathscr{S}$ is Bernoulli, its associated time-1 action $\left\{S_{\mathrm{v}}\right\}_{\mathbf{v} \in \mathbb{Z}^{n}}$ is also Bernoulli. By the Classification Theorem, the finite extension $\left\{S_{v}^{g}\right\}_{\mathbf{v} \in \mathbb{Z}^{n}}$ (the time-1 action of $\mathscr{S}^{g}$ ) either is Bernoulli or is not weakly mixing. If $\left\{S_{v}^{g}\right\}_{v} \in \mathbb{Z}^{n}$ is Bernoulli, then so too is the flow $\mathscr{S}^{g}$.

Suppose, instead, that $\left\{S_{v}^{g}\right\}_{v} \in \mathbb{Z}^{n}$ is not weakly mixing. According to the classification outlined above, there exist disjoint sets $A_{1}, \ldots, A_{m}$ which partition $\widehat{X}, m \leq k$, such that each $S_{\mathrm{v}}^{g}$ acts as a permutation on the $A_{i}$ 's. These $A_{i}$ 's generate the invariant algebra for $\left\{S_{v}^{g}\right\}_{\mathbf{v} \in \mathbb{Z}^{n}}$. Let $\mathbf{t} \in \mathbb{R}^{n}$. Since each $S_{\mathrm{t}}^{g}$ commutes with the action $\left\{S_{\mathrm{v}}^{g}\right\}_{\mathrm{v} \in \mathbb{Z}^{n}}$, each $S_{\mathrm{t}}^{g}$ must also act as a permutation on $\left\{A_{1}, \ldots, A_{m}\right\}$. However, $\mathscr{S}^{g}$ is a measurable flow, so that $\lim _{|\mathfrak{t}| \rightarrow 0} \hat{\mu}\left(S_{\mathbf{t}}^{g}\left(A_{1}\right) \triangle A_{1}\right)=0$. Hence there exists a neighborhood $U$ of 0 (in $\mathbb{R}^{n}$ ) such that $S_{\mathbf{t}}^{g}\left(A_{1}\right)=A_{1}, \forall \mathrm{t} \in U$. Since $\mathbb{R}^{n}$ is a group, this implies that $A_{1}$ is an invariant set for all $S_{\mathrm{t}}^{g}, \mathbf{t} \in \mathbb{R}^{n}$. Hence $\mathscr{S}^{g}$ is a nonergodic flow. Even more, each $A_{i}$ is invariant under the flow, but, when restricted to a particular $A_{i}$, the flow is actually Bernoulli.

The techniques used here are being generalized to classify the compact extensions of a $\mathbb{Z}^{n}$-Bernoulli shift. This work is currently underway.

\section{REFERENCES}

1. J. W. Kammeyer, A complete classification of the two-point extensions of a multidimensional Bernoulli shift, J. Analyse Math. 54 (1990), 113-164.

2. _ The isomorphism theorem for relatively finitely determined $\mathbb{Z}^{n}$-actions, Israel J. Math. 69 (1990), 117-127.

3. D. J. Rudolph, If a finite extension of a Bernoulli shift has no finite rotation factors, then it is Bernoulli, Israel J. Math. 30 (1978), 193-206.

4. __ Counting the relatively finite factors of a Bernoulli shift, Israel J. Math. 30 (1978), 255-263.

5. __ Classifying the isometric extensions of a Bernoulli shift, J. Analyse Math. 34 (1978), 36-60.

6. _ An isomorphism theory for Bernoulli free $\mathbb{Z}$-skew-compact group actions, Adv. in Math. 47 (1983), 241-257.

Department of Mathematics, United States Naval Academy, 572 Holloway Road, ANNAPOLIS, MARYLAND 21402-5002

E-mail address: jwk@math2.sma.usna.navy.mil 